\title{
A Technical Study on Cooperative Structure of a Hybrid Network for Video Content Delivery
}

\author{
Diego Pajuelo \\ Yuzo Iano \\ Paulo E. R. Cardoso \\ Raphael O. Barbieri \\ Daniel Izario \\ Euclides Lourenço Chuma \\ Silvio M. Carvalho
}




\title{
A Technical Study on Cooperative Structure of a Hybrid Network for Video Content Delivery
}

\author{
Diego Pajuelo, Yuzo Iano, Member, IEEE, Paulo E. R. Cardoso, Raphael O. Barbieri, Daniel Izario, \\ Euclides Lourenço Chuma, Silvio M. de Carvalho
}

\begin{abstract}
Cooperative structure of hybrid network is a topic of current interest in academia and industry since can serve to enhance content delivery technologies in order to face the "mobile data tsunami". This paper considers the latest technologies either in the broadcast and broadband environment to encourage the use of flexible bootstrap in the physical layer.
\end{abstract}

Index Terms - Broadcasting, Broadband, Television System.

\section{INTRODUCTION}

$\mathrm{N}$ OWADAYS, broadband networks have experimented many changes because exponential growth of media consumption. For instance, Internet traffic in Latin America will expect to reach 12.9 exabytes, comparable to 3 billion of DVD, per month in 2019 [1]. On-demand and streaming services have become predominant for users by offering rich interaction and a vast offer of audiovisual content.

Video delivery in mobile devices is a challenging task due to unexpected increment of users along the last years in broadband networks, both in local and cellular networks. For this reason, a robust and efficient video content delivery towards mobile users have attracted so much interest by academic and enterprise community. Mobile and broadcast industry have reacted by proposing new Information and Communication Technology (ICT) in order to improve the television viewing experience in small devices and to offer better multimedia services. Delivering high-quality video mobile services to indoor, portable and hand-held receivers is challenging because of high-noise levels in wireless communication channels, fading and doppler effect.

Historically, broadcasting and broadband technologies have walked on separate railroads, building its own infrastructure and business model. It was thought that these technologies were incompatibles regarding the type of services they delivered. Nevertheless, the growing demand for audiovisual content by users from all over the world paves the way for a cooperation in terms of infrastructure and a converged transport architecture.

The Fifth Generation Mobile Networks, 5G, open countless opportunities for convergence of mobile broadband and broadcast services. For instance, it is expected that transmission of mass multimedia services to mobile and stationary receivers through different network infrastructures might be allowed and the radio functions can be configured on demand depending on the specific needs of the service and the status of the network [2]. Besides that, $5 \mathrm{G}$ requirements point out that can handle enormous numbers of connected devices; because of the advent of new technologies such as Internet of Things
(IoT), vehicle to vehicle (V2V), machine to machine (M2M) and device to device (D2V) communication, and a latency of 1 $\mathrm{ms}$, almost ten times reduction from 4G's [3]. For that reason, optimized and efficient video mobile traffic offloading is of high relevance for future communication platforms.

Additionally, the advent of new technologies, such as the $4 \mathrm{~K}$ ultra-high definition television (UHDTV), the high dynamic range (HDR), the higher frame rate and the wider color gamut, allows to user experiencing wider range of services; Virtual reality (VR), augmented reality (AR), Free ViewPoint Television (FVT) and Multi/Companion Screen Viewing are becoming increasingly popular and will be offered to smart devices in the coming years.

This paper aims at investigating how beneficial could be a converged broadband/broadcast network regarding to current technologies have been deployed. Also, is detailed each layer of the network where can work jointly and in complementary way in order to get a more efficient content delivery over the core and radio access network.

The remaining of this paper is organized as follows: Section II presents a technical overview of the system model. Section III proposes the cooperative structure of the video content delivery network. Finally, Section IV concludes this paper.

\section{System ModeL}

People search for new audiovisual services anywhere and at any time. They are rarely aware of the radio access technology they are using when connecting to broadband or broadcast network. Personalized media experiences are demanded because of providing better quality of experience (QoE) to the users [4]. Hence, future network solutions must absorb as much as possible of field experience from latest content delivery standards that have been deployed in different countries in the last years in order to meet the peoples's demand and consumer behaviour about how the appearing of new technologies can impact in their everyday activities. For instance, pure broadcast solutions in the mobile ecosystem did not attract the attention of users when were launched because that technology only worked in specific devices. The lesson learned was that a mobile broadcast standard must be supported by a worldwide ecosystem to promote continuous development, that is of crucial importance to market success [5].

The new challenges for future networks is to provide higher data capacity, more robust performance, better spectrum efficiency with lower power consumption and CAPEX/OPEX cost. New technologies must enable deep indoor TV services, 
mobile services and traditional TV services, using a fixed reception outdoor over-roof antenna. Furthermore, it is important to take into account the different scenarios, either urban, suburban or rural in order to envisage the technical details of infrastructure and physical layer of the network.

Advantages and disadvantages of main technologies of video delivery over wireless channels will be considered, since the system model is based on a thorough review of the state-of-the-art of these technologies that give solution to different technical problems that this work tries to solve. From this point of view, is important to point out that there exist no elements to suggest that technologies involved in mobile networks are better than technologies from broadcasting. This work tries to explore and highlight the importance of seeking synergy between the two networks in benefit of future media transmission.

To cope with the unlimited levels of demand at peak times in densely populated areas, broadcasting is the ideal solution for urban scenarios, however, broadcast efficiency decreases when broadcasting less popular TV channels [6], a smart dynamic TV scheduling framework is required to maximize radio resource usage [7]. On the other hand, mobile networks have been evolving along the years from first transmitting voice services to delivering high quality video content in mobility environment. Despite of this, people prefer to reserve higher bandwidth activities for the home or workplaces by connecting to local wireless connections, engaging lower bandwidth services while is stayed outside [8], because of high data plan cost. Unlimited internet access over mobile networks are offered by operators in United States, pursuing the growing demand of mobile video. This is one the key factors driving the wireless industry to develop the fifth generation of network technology [9].

The system model should comply fundamental requirements to make feasible an efficient content delivery over hybrid network aiming at creating a revolutionary and disruptive audiovisual medium that enables the best user experience and can be the starting point for a global integration in terms of development and cooperation, thinking out beyond 2020 . Therefore, then main requirements are: to form a common physical [5] and transport layer, to develop a return channel among users and service providers, to make flexible and efficient use of the available spectrum; and to provide system forward compatibility. To comply with these requirements, this work will emphasize on how cutting-edge technologies that have been deployed in recent years contribute in the sketching of the final network infrastructure.

The latest video content delivery standards over broadcast and broadband network have improved channel robustness, get better spectral efficiency and higher data capacity (throughput) thanks to developing new physical interface technologies. As a unified physical interface for wireless communications, OFDM modulating waveform is used to cope with time and frequency selective channels. Also, physical layer framing carries signal basic information about the technology in a way that the type of service can be described. Concerning the transport layer, the use of Internet Protocol (IP) instead of Transport Stream (TS) opens new opportunities for development of routing protocols
[10] aiming at creating a single communication network, involving external networks, either a local network such as WiFi or a mobile network. Carrier Aggregation (CA) approach allows to combine different carries to a virtual channel and thereby to increase both bandwidth and data rate. One carrier may be configured as a broadcasting service and in the same way be connected to a cellular network simultaneously [11].

\section{Video Content Delivery OVer a CoOperative STRUCTURE NETWORK}

In this section, the main technologies that support and make viable the proposal of this work will be addressed and will be explained the reasons they were chosen. Our approach involves the exhaustive revision of theoretical framework from baseline technologies of physical layer, transport layer and application layer to software-based technologies, whose main ideas may beneficial with the mission of sketching, building and deploying a future cooperative structure network.

\section{A. Physical Layer Baseline Technology}

The emergence of a new multiplexing technology that takes advantage of time, frequency and signal strength in a interval time dramatically improves spectral efficiency. Layered Division Multiplexing (LDM) [12] is a constellation superposition technology, controlled by power level differences of two data streams, known as Upper Layer (UL) and Lower Layer (LL). Figure 1 shows the LDM 2-Layer Transmitter and Figure 2 shows the LDM 2-Layer Transmitter, these figures were extracted from [13].

LDM was adopted by ATSC 3.0 [14] as one of the physical layer baseline technologies and is investigated as an enabling technology for future broadband (LTE or 5G) systems to deliver multicast/broadcast services with high spectrum efficiency [13].

The LDM receiver, depicted in Figure 2, can only decode the strongest signal layer to allow certain backward compatibility with existing technologies, whereas cutting-edge devices can decode signal with lower power levels. For instance, coexistence of ISDB-Tb and new technologies could be deployed by performing a two-layer LDM transmission. This ensures a soft transition towards hybrid network solutions. Moreover, experimental results demonstrated that this arrangement can transmit more data and be more robust against noise and multipath interference using reception diversity [15]. LDM can coexist with emerging technologies, such as Bit Interleaved and Coded Modulation (BICM) to configure a more robust signal to delivery high data rate services such as UHDTV, tailored to fixed reception, and robust indoor/hand-held/mobile services over a large coverage area, strong channel coding and modulated is mandatory to provide good detection at very low SNR in the same spectrum resource [12]

Figure 3 depicts the block diagram of the BICM module. Its main importance is the wide range of baseband resource tools, such as Non-Uniform constellations (NU-QAM) and 24 original low-density parity check (LDPC) codes with coding rates from $2 / 15(0.13)$ up to $13 / 15(0.87)$ for two code sizes: 16 200 bits and 64800 bits. It is though to achieve high efficiency 


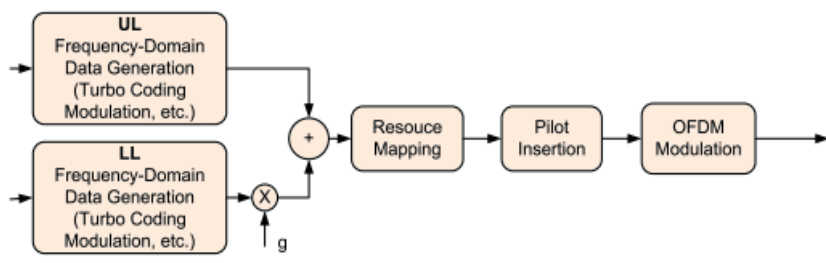

Fig. 1. LDM 2-Layer Transmitter

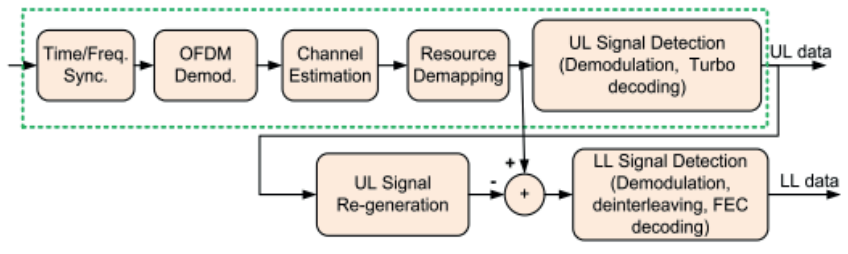

Fig. 2. LDM 2-Layer Receiver

over a wide range of reception conditions. Concerning to performance, the spectral efficiency is closer to the theoretical Shannon limit [16]. Channel bonding is a good alternative to increase spectrum flexibility for broadcasting with the possibility to provide scalable video coding services across two Radio Frequency (RF) channels [17]. Free ViewPoint Television (FVT) and Multi/Companion Screen Viewing could be offered by using this type of technology.

Tower overlay over LTE-Advanced $+(\mathrm{TOoL}+)$ provides an interesting tool for converged network approaches, taking advantage of traditional broadcasting services that works over a High Tower High Power (HTHP) infrastructure in order to cover large areas at low cost, reducing power consumption. Besides this, this approach can cope with reduced Inter-Site Distance (ISD) of towers in cell network due to short OFDM cyclic prefix mode allowing larger Single Frequency Networks (SFN) and overcome the main drawback of evolved Multimedia Broadcast Multicast Services (eMBMS), the shared spectrum with unicast services in Time Division Multiplexing (TDM) which almost occupies $60 \%$ of bandwidth resources [18]. Figure 4 presents the basic diagram of the technology, the figure was extracted from [11].

With the development and deployment of the technologies described above, cognitive radio access networks, involving cooperative broadband and broadcast solutions can handle consumption of video content from a large audience. For example, transmission of sporting events, which are mostly consumed live and have little interest for time-shifted viewing, can switch radio transmission between broadband and broadcast access, optimizing the total data rate used by the network, that would be transparent for end user.

\section{B. Transport Layer Baseline Technology}

For many years, TS was the anchor transport layer protocol since the advent of digital television. Principally, because of constant delay across the delivery link between users and station; and that the streams could be encrypted by packet. Conversely, growth of content delivery services are based on

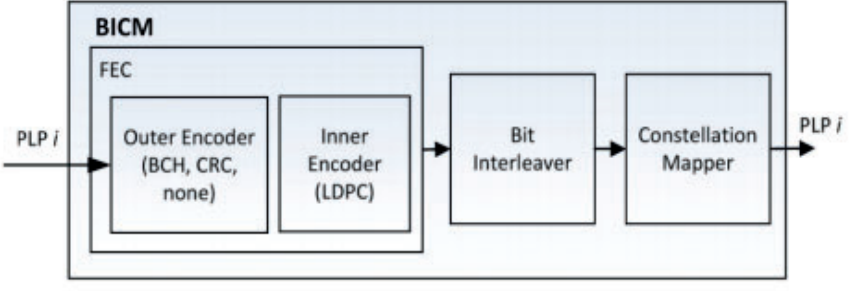

Fig. 3. Block diagram of the BICM module

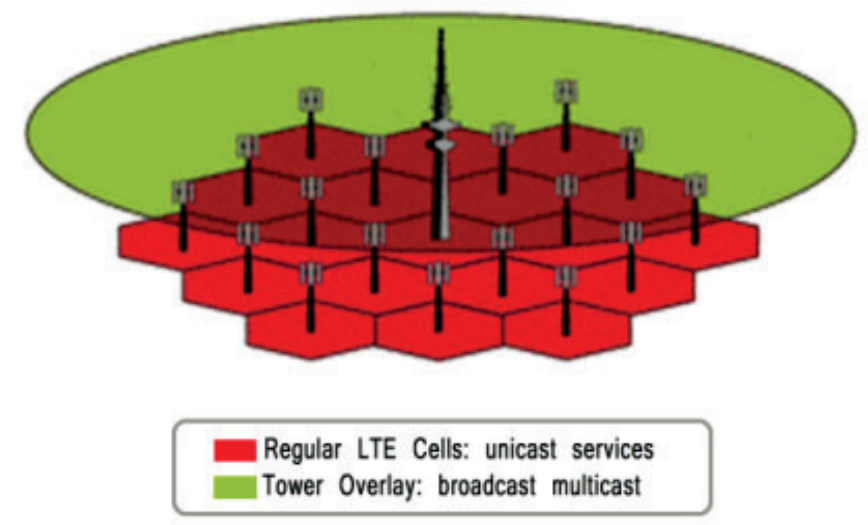

Fig. 4. The Tower Overlay Overlay over LTE-A+

HTTP Streaming, where the deployment of servers and caches became easy, economic, and scalable. For this reason, IP stack is fundamental for user interactivity and to get better user experience [10]. Figure 5 shows the unified broadcast/broadband IP Stack. This allows to developers using stable version of software frameworks such as HTML5 and JavaScript (JS).

\section{Cooperative Structure Network}

This work encourages the use of a Unified Broadcast Layer (UBL) for delivery of multimedia services. Technologies such as LDM, BICM and channel bonding should be part of the physical framing, however is important the creating of a new bootstrap,that provides synchronization and signals basic information about the technology used in the physical layer itself, this is important because new advances with respect to multiplexing, modulation or LDPC codes in the future can be added with no restriction.

The development and deployment of this cooperative structure should be on software-based technologies such as Cloud Radio Access Network (C-RAN), Software-defined networking (SDN)-based framework and Network Function Virtualization (NFV) approaches. these technologies are promising and envision more democratic platforms, making the telecommunications sector more free.

\section{CONCLUSion}

The main contribution of this work is to highlight the importance of hybrid transmission structure on video-content 


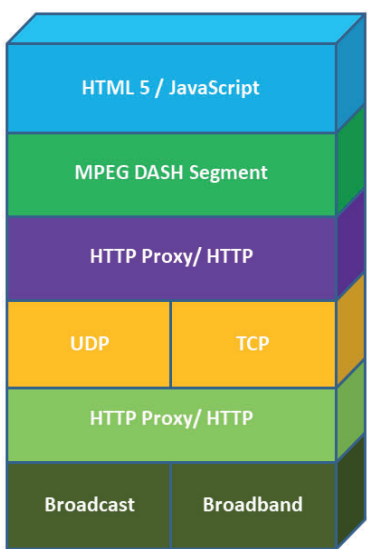

Fig. 5. IP Stack

delivery solution. Latest Broadband and Broadcast technologies can be crucial to enhance in terms of efficiency the future wireless communications and will provide a better user experience.

\section{ACKNOWLEDGMENT}

The authors would like to thank the CAPES (Coordenação de Aperfeiçoamento de Pessoal de Nível Superior) and FAEPEX (Fundo de Apoio ao Ensino, à Pesquisa e Extensão) programs for the financial support and the academic incentive.

\section{REFERENCES}

[1] White Paper, "Cisco visual networking index: Forecast and methodology, 2014-2019," Cisco, 2015. [Online]. Available: www.cisco.com

[2] J. Calabuig, J. F. Monserrat, and D. Gomez-Barquero, "5th generation mobile networks: A new opportunity for the convergence of mobile broadband and broadcast services," IEEE Communications Magazine, vol. 53, no. 2, pp. 198-205, 2015.

[3] M. Agiwal, A. Roy, and N. Saxena, "Next Generation 5G Wireless Networks: A Comprehensive Survey," IEEE Communications Surveys Tutorials, vol. 18, no. 3, pp. 1617-1655, 2016.

[4] F. Boronat, M. Montagud, D. Marfil, and C. Luzon, "Hybrid Broad-cast/ Broadband TV Services and Media Synchronization: Demands, Preferences and Expectations of Spanish Consumers," IEEE Transactions on Broadcasting, vol. 64, no. 1, pp. 52-69, 2018.

[5] M. Crussiere, C. Douillard, C. Gallard, M. Le Bot, B. Ros, A. Bouttier, and A. Untersee, "A unified broadcast layer for horizon 2020 delivery of multimedia services," IEEE Transactions on Broadcasting, vol. 60, no. 2, pp. 193-207, 2014

[6] C. P. Lau and B. Shihada, "TV broadcast efficiency in $5 \mathrm{G}$ networks from subscriber prospective," 2015 IEEE Global Communications Conference, GLOBECOM 2015, pp. 0-5, 2015.

[7] C. P. Lau, A. Alabbasi, and B. Shihada, "An Efficient Live TV Scheduling System for 4G LTE Broadcast," IEEE Systems Journal, vol. 11, no. 4, pp. 2737-2748, 2017.

[8] S. Lax and M. Ala-Fossi, "The Short Future of Public Broadcasting Replacing DTT with IP ?" International Communication Gazzete, pp. 365-382, 2016.

[9] J. Montalban, P. Scopelliti, M. Fadda, E. Iradier, C. Desogus, P. Angueira, M. Murroni, and G. Araniti, "Multimedia Multicast Services in 5G Networks: Subgrouping and Non-Orthogonal Multiple Access Techniques," IEEE Communications Magazine, vol. 56, no. 3, pp. 96-103, 2018.

[10] G. K. Walker, T. Stockhammer, G. Mandyam, Y.-K. Wang, and C. Lo, "ROUTE/DASH IP Streaming-Based System for Delivery of Broadcast, Broadband, and Hybrid Services," IEEE Transactions on Broadcasting, vol. 62 , no. 1, pp. 328-337, mar 2016.
[11] D. Rother, S. Ilsen, and F. Juretzek, "A Software Defined Radio based Implementation of the "Tower Overlay over LTE-A+" System," 2014 IEEE International Symposium on Broadband Multimedia Systems and Broadcasting, pp. 1-6, 2014.

[12] L. Zhang, W. Li, Y. Wu, X. Wang, S. I. Park, H. M. Kim, J. Y. Lee, P. Angueira, and J. Montalban, "Layered-Division-Multiplexing: Theory and Practice," IEEE Transactions on Broadcasting, vol. 62, no. 1, pp. 216-232, 2016.

[13] L. Zhang, Y. Wu, G. K. Walker, W. Li, K. Salehian, and A. Florea, "Improving LTE \{e\} MBMS with extended OFDM parameters and layereddivision-multiplexing," IEEE Transactions on Broadcasting, vol. 63 , no. 1 , pp. 32-47, 2017.

[14] R. Chernock, D. Gómez-Barquero, J. Whitaker, S. I. Park, and Y. Wu, "ATSC 3.0 Next Generation Digital TV Standard - An Overview and Preview of the Issue," IEEE Transactions on Broadcasting, vol. 62, no. 1, pp. 154-158, 2016.

[15] R. Rabaça, C. Akamine, G. de Oliveira, and T. Sapia, "Robustness against the effects of multipath in an ISDB-T LDM broadcast system using diversity at reception," SET International Journal of Broadcast Engineering, vol. 2017, no. 3, pp. 37-43, nov 2017.

[16] L. Michael and D. Gomez-Barquero, "Bit-Interleaved Coded Modulation (BICM) for ATSC 3.0," IEEE Transactions on Broadcasting, vol. 62, no. 1, pp. 181-188, mar 2016.

[17] L. Stadelmeier, D. Schneider, J. Zollner, and J. J. Gimenez, "Channel Bonding for ATSC3.0," IEEE Transactions on Broadcasting, vol. 62, no. 1, pp. 289-297, mar 2016.

[18] S. Ilsen, F. Juretzek, L. Richter, D. Rother, and P. Bretillon, "Tower overlay over LTE-Advanced+ (TOoL+): Results of a field trial in Paris," in 2016 IEEE International Symposium on Broadband Multimedia Systems and Broadcasting (BMSB) IEEE, jun 2016, pp. 1-6.

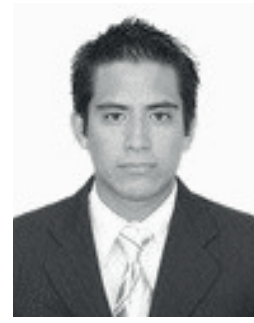

Diego Pajuelo Graduate in Electrical Engineering from the Peruvian University of Applied Sciences (UPC), Lima, Peru in 2012. He is currently working towards his Doctoral degree in Sciences and Telecommunications at Unicamp. His research interests are: HDR Video and audio coding, Image processing, Digital television and Satellite communications.

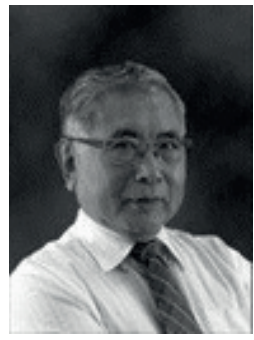

Yuzo Iano is the head and founder of the LCV since 1972. He obtained his BSc (1972), MSc (1974) and $\mathrm{PhD}$ (1986) in Electrical Engineering at Unicamp, SP-Brazil. Research Interests: Digital Signal Processing (images/audio/video), Digital TV, 4G (LTE) and $5 \mathrm{G}$ Cellular Networks, Pattern Recognition, Smart Cities, Smart Grid, Internet of Things.

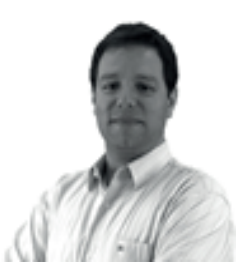

Paulo E. R. Cardoso Holds a degree in Electrical Engineering from the FEEC- UNICAMP (2002) and a MSc degree in Electrical Engineering (Electronics) by DEMIC-FEEC-UNICAMP (2005). He is currently a $\mathrm{PhD}$ candidate in the LCV- DECOM-FEECUNICAMP, searching Digital TV. Licensed from the post of Specialist in Regulating in the Agência Nacional de Telecomunicações - Anatel, where it operates in coordination of grants and resources to the provision, working with the licensing and amendment of technical characteristics of broadcasting stations. Previously, he served in the surveillance in broadcasters and in the solution of problems of radio interference in any system of telecommunication. He was responsible for the Technical Regulation to Broadcasting in Modulated Frequency and analysis of processes of technical feasibility for inclusion or amendment of the Basic Plan of Distribution Channels of Broadcasting in Modulated Frequency. He participated as an observer in the Federal Government in testing of Digital Radio Broadcasting, both in tests of American Standard -HD Radio, in 2008 and 2012, as in tests of the European standard - DRM, in 2010. He has worked as a telecommunications researcher of the Fundação Centro de Pesquisas e Desenvolvimento - CPqD. 

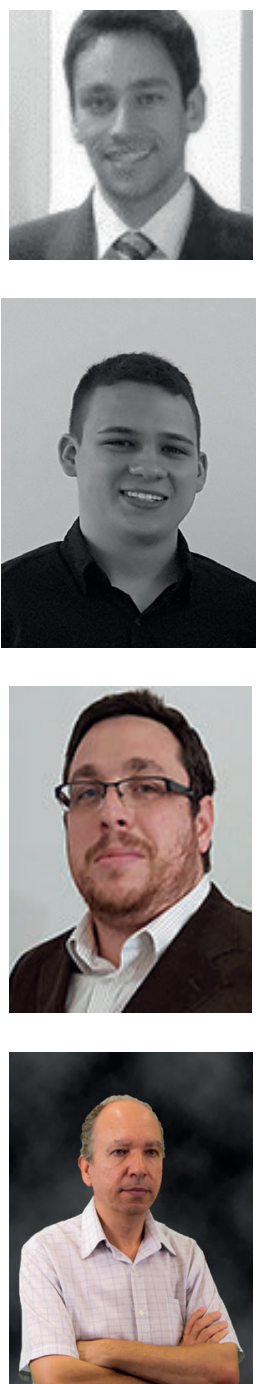

Raphael O. Barbieri Graduated in Computer Engi-neering from FEEC- UNICAMP (2007) and MBA in Project Management from IBE-FGV (2014). Mem-ber of Technical Module of SBTVD Forum and Product Manager at EiTV. He is currently working on his Master's degree in Electrical Engineering at FEEC-Unicamp. Research Interests: Digital TV, ISDB-Tb and Broadcast Engineering.

Daniel Izario Bachelor's at National Institute of Telecommunications/MG, Brazil - Inatel in Computer Engineering (2017), master's degree student at Computer Engineering from State University of Campinas. He is currently a freelancer in the development and planning of websites for stores and businesses and personal websites. His research interests are digital transmission and image/video/data processing, javascript, facial recognition, discrete cosine transform, data transmission and storage, defense systems and smart cities.

Euclides Lourenço Chuma earned a degree in Mathematics from UNICAMP (2003), graduate degree in Network and Telecommunications Systems in the INATEL (2015), and MSc in Electrical Engineering at UNICAMP (2017). Currently is $\mathrm{PhD}$ Candidate in Electrical Engineering at UNICAMP, SP-Brazil. His research interests are Antennas, Microwave, Millimeter-Wave, Wireless Power Transfer, Software Defined Radio and Cognitive Radio.

Silvio Renato Messias de Carvalho has $\mathrm{PhD}$ de-gree, MSc degree, electrical engineer and occupa-tional safety engineer degrees, from Unicamp. He has professional experience in the areas of video and audio, radiating systems, RF transmission and reception systems as well as power infrastructure, critical mission and alternative energies. 